\title{
Solvability of an Operator Riccati Integral Equation in a Reflexive Banach Space
}

\author{
Nikita Artamonov ${ }^{* \dagger}$ \\ ${ }^{\dagger}$ MGIMO University
}

June 24, 2019

\begin{abstract}
We show that if $X$ is a reflexive Banach space, then a nonautonomous operator Riccati integral equation has a unique strongly continuous self-adjoint nonnegative solution $P(t) \in \mathcal{L}\left(X, X^{*}\right)$
\end{abstract}

\section{Preliminaries}

It is well known $[1,2,3,4,5]$, that the solution of a linear-quadratic control problem on a finite interval can be expressed via the solution of an operator Riccati (differential or integral) equation considered in the space of operator functions.

Some results on the solvability of autonomous and nonautonomous Riccati equations in operator functions ranging in the space $\mathcal{L}(\mathcal{H})$ where $\mathcal{H}$ is a Hilbert space, were obtained in $[1,3,6,7]$ and $[2,4]$, respectively.

A triple $X \hookrightarrow \mathcal{H} \hookrightarrow X^{*}$ of spaces with dense embeddings was considered in [5] for a Hilbert space $X$ and in [8] for a reflexive Banach space $X$. In these papers, the solvability of an autonomous Riccati equation in operator functions ranging in the spaces $\mathcal{L}\left(X^{*}, X\right)$ and $\mathcal{L}\left(X, X^{*}\right)$, respectively, was established. In the papers [8,9], the solvability of the Riccati equation

*nikita.artamonov@gmail.com 
was used to prove the solvability of systems of forward-backward evolution equations.

The present paper generalizes the above-mentioned results. We prove that there exists a unique solution of the Riccati integral equation for strongly continuous operator functions ranging in the space $\mathcal{L}\left(X, X^{*}\right)$, where $X$ is an arbitrary reflexive Banach space. It is important to note that, in contrast to the papers $[8,5]$, we do not assume an embedding between the space $X$ and the dual space.

1.1 By $\mathcal{L}\left(X_{1}, X_{2}\right)$ we denote the normed space of continuous linear operators from a Banach space $X_{1}$ to a Banach space $X_{2}$. Just as in [1, Part IV], we introduce the following spaces of operator functions. By $C_{u}\left([a, b] ; \mathcal{L}\left(X_{1}, X_{2}\right)\right)$ we denote the Banach space of strongly continuous operator functions on the interval $[a, b]$ ranging in $\mathcal{L}\left(X_{1}, X_{2}\right)$ with the norm

$$
\|P\|_{u}=\sup _{t \in[a, b]}\|P(t)\|_{\mathcal{L}\left(X_{1}, X_{2}\right)} .
$$

and by $C_{s}\left([a, b] ; \mathcal{L}\left(X_{1}, X_{2}\right)\right)$ we denote the topological space of strongly continuous operator functions on $[a, b]$ ranging in $\mathcal{L}\left(X_{1}, X_{2}\right)$ with the topology of uniform strong convergence. By definition, $P \in C_{s}\left([a, b] ; \mathcal{L}\left(X_{1}, X_{2}\right)\right)$ if and only if the vector function $(P x)(t)=P(t) x$ belongs to the Banach space $C\left([a, b] ; X_{2}\right)$ for each $x \in X_{1}$. If $X_{1}=X_{2}=Y$, then we write $C_{s}([a, b] l ; \mathcal{L}(Y))$ instead of $C_{s}([a, b] ; \mathcal{L}(Y, Y))$. Note that if $P \in C_{s}\left([a, b] ; \mathcal{L}\left(X_{1}, X_{2}\right)\right)$, then the function $\|P(\cdot)\|_{\mathcal{L}\left(X_{1}, X_{2}\right)}$ is measurable and bounded and the function $\|P(\cdot) x\|_{X_{2}} \in C[a, b]$ for each $x \in X_{1}$. By definition, a sequence $\left\{P_{k}\right\}_{k=1}^{+\infty}$ converges to $P$ in the space $C_{s}\left([a, b] ; \mathcal{L}\left(X_{1}, X_{2}\right)\right)$ if and only if the sequence of vector functions $P_{k} x$ converges to the vector function $P x$ uniformly on $[a, b]$ (i.e., $P_{k} x$ converges to $P x$ in space $C\left([a, b] ; X_{2}\right)$ ) for each $x \in X_{1}$. A straightforward verification shows that if $P \in C_{s}\left([a, b] ; \mathcal{L}\left(X_{1}, X_{2}\right)\right)$ and $Q \in C_{s}\left([a, b] ; \mathcal{L}\left(X_{2}, X_{3}\right)\right)$, then $Q P \in C_{s}\left([a, b] ; \mathcal{L}\left(X_{1}, X_{3}\right)\right)$.

The topological space of strongly continuously differentiable operator functions $C_{s}^{1}\left([a, b] ; \mathcal{L}\left(X_{1}, X_{2}\right)\right)$ with the topology of uniform strong convergence is defined in a similar way. By definition, $P \in C_{s}^{1}\left([a, b] ; \mathcal{L}\left(X_{1}, X_{2}\right)\right)$ if and only if the vector function $P x$ belongs to space $C^{1}\left([a, b] ; X_{2}\right)$ for each $x \in X_{1}$.

Throughout the paper, slim stands for the limit in the strong operator topology; for convenience, we denote the interval $\mathcal{I}=[0, T]$.

1.2 Let $X_{1}$ and $X_{2}$ be Banach spaces, and let operators $A_{1} \in \mathcal{L}\left(X_{1}\right)$, $A_{2} \in \mathcal{L}\left(X_{2}\right), C, G \in \mathcal{L}\left(X_{1}, X_{2}\right)$ and $B \in \mathcal{L}\left(X_{2}, X_{1}\right)$ be given. Since the 
operators $A_{1}$ and $A_{2}$ are bounded, it follows that they are the generators of $C_{0}$-groups $e^{t A_{i}} \in \mathcal{L}\left(X_{1,2}\right)(t \in \mathbb{R}), i=1,2$.

In the collection of spaces $\left(X_{1}, X_{2}\right)$, consider the autonomous backward (in time) Riccati differential equation

$$
P^{\prime}(t)=-C+A_{2} P(t)+P(t) A_{1}-P(t) B P(t) \quad P(T)=G .
$$

on interval $\mathcal{I}$. A straightforward verification shows that if operator function $P \in C_{s}^{1}\left(\mathcal{I} ; \mathcal{L}\left(X_{1}, X_{2}\right)\right)$ is a solution of this equation, then the operator function $P$ satisfies the integral equation

$$
P(t)=e^{(t-T) A_{2}} G e^{(t-T) A_{1}}+\int_{t}^{T} e^{(t-r) A_{2}}(C-P(r) B P(r)) e^{(t-r) A_{1}} d r,
$$

where integral is understood in the strong sense. This equation can be called an autonomous Riccati integral equation.

Let $Y$ be a Banach space.

Definition 1. An operator function $\left\{U_{t, s}\right\}_{0 \leq s \leq t \leq T} \subset \mathcal{L}(Y)$ is called forward (in time) evolution family in $\mathcal{L}(Y)$ if it has the following properties:

1. The relation $U_{s, s}=I_{Y}$ holds for each $s \in[0, T]$;

2. The relation $U_{t, s}=U_{t, r} U_{r, s}$ holds for each $0 \leq s \leq r \leq t \leq T$.

Definition 2. An operator function $\left\{V_{t, s}\right\}_{0 \leq t \leq s \leq T} \subset \mathcal{L}(Y)$ is called a backward (in time) evolution family in $\mathcal{L}(Y)$ if it has the following properties:

1. The relation $V_{s, s}=I_{Y}$ holds for each $s \in[0, T]$;

2. The relation $V_{t, s}=V_{t, r} V_{r, s}$ holds for each $0 \leq t \leq r \leq s \leq T$.

Remark 1. It readily follows from these definitions that if $U_{t, s}$ is a forward evolution family in $\mathcal{L}(Y)$, then $V_{\tau, \sigma}=U_{\sigma, \tau}^{*}$ is a backward evolution family in $\mathcal{L}\left(Y^{*}\right)$.

Definition 3. A (forward or backward) evolution family $U_{t, s}$ is said to be strongly continuous if it is strongly continuous in $t$ and s separately, i.e., strongly continuous in $t$ for each $s$ and in $s$ for each $t$.

Remark 2. A strongly continuous (forward or backward) evolution family is not necessarily jointly strongly continuous in $(t, s)$. Moreover, it may not be even uniformly bounded in the operator norm [4, Appendix B] 
Remark 3. In what follows, we conveniently use arrows to indicate forward and backward evolution families; namely, we write $\overleftarrow{U}_{t, s}$ and $\vec{U}_{t, s}$ respectively.

Definition 4. Let $\overleftarrow{U}_{t, s}$ be a strongly continuous forward evolution family in $\mathcal{L}\left(X_{1}\right)$, let $\vec{U}_{t, s}$ be a strongly continuous backward evolution family in $\mathcal{L}\left(X_{2}\right)$ and assume that

$$
C \in C_{s}\left(\mathcal{I} ; \mathcal{L}\left(X_{1}, X_{2}\right)\right), \quad B \in C_{s}\left(\mathcal{I} ; \mathcal{L}\left(X_{2}, X_{1}\right)\right), \quad G \in \mathcal{L}\left(X_{1}, X_{2}\right) .
$$

The integral equation

$$
P(t)=\vec{U}_{t, T} G \overleftarrow{U}_{T, t}+\int_{t}^{T} \vec{U}_{t, r}\{C(r)-P(r) B(r) P(r)\} \overleftarrow{U}_{r, t} d r
$$

for an operator function $P \in C_{s}\left(\mathcal{I} ; \mathcal{L}\left(X_{1}, X_{2}\right)\right)$ will be called the backward (in time) Riccati integral equation with the condition $P(T)=G$ in the collection of spaces $\left(X_{1}, X_{2}\right)$. The integral is understood in the strong sense.

Remark 4. It follows from Definition 1 and the semigroup property of evolution families that if $P \in C_{s}\left(\mathcal{I} ; \mathcal{L}\left(X_{1}, X_{2}\right)\right)$ is a solution of the Riccati integral equation (1), then the relation

$$
P(t)=\vec{U}_{t, \tau} P(\tau) \overleftarrow{U}_{\tau, t}+\int_{t}^{\tau} \vec{U}_{t, r}\{C(r)-P(r) B(r) P(r)\} \overleftarrow{U}_{r, t} d r
$$

holds for all $0 \leq t \leq \tau \leq T$.

1.3 The following result for a Banach space $Y$ is well known [10, Theorem $9.19]$

Theorem 1. Let $\overleftarrow{U}_{t, s}$ be a strongly continuous uniformly bounded forward evolution family in $\mathcal{L}(Y)$, and let $Q \in C_{s}(\mathcal{I} ; \mathcal{L}(Y))$. Then there exists a unique strongly continuous uniformly bounded forward evolution family $\overleftarrow{\Psi}_{t, s}$ in $\mathcal{L}(Y)$, satisfying the equations $(0 \leq s \leq t \leq T)$

$$
\begin{aligned}
& \overleftarrow{\Psi}_{t, s}=\overleftarrow{U}_{t, s}+\int_{s}^{t} \overleftarrow{U}_{t, r} Q(r) \overleftarrow{\Psi}_{r, s} d r \\
& \overleftarrow{\Psi}_{t, s}=\overleftarrow{U}_{t, s}+\int_{s}^{t} \overleftarrow{\Psi}_{t, r} Q(r) \overleftarrow{U}_{r, s} d r
\end{aligned}
$$


A similar result is true for strongly continuous uniformly bounded backward evolution families.

Let us show that the family $\overleftarrow{\Psi}_{t, s}$ continuously depends on the operator function $Q$.

Proposition 1. Let $\overleftarrow{U}_{t, s}$ be a uniformly bounded strongly continuous forward evolution family in $\mathcal{L}(Y)$, and let a sequence $\left\{Q_{n}\right\}_{n=1}^{+\infty}$ of operator functions converge to $Q$ in the space $C_{s}(\mathcal{I} ; \mathcal{L}(Y))$. Further, let strongly continuous forward evolution families $\overleftarrow{\Psi}_{t, s}^{(n)}$ and $\overleftarrow{\Psi}_{t, s}$ in $\mathcal{L}(Y)$ be solutions of the equations $(0 \leq s \leq t \leq T)$

$$
\begin{aligned}
& \overleftarrow{\Psi}_{t, s}^{(n)}=\overleftarrow{U}_{t, s}+\int_{s}^{t} \overleftarrow{U}_{t, r} Q_{n}(r) \overleftarrow{\Psi}_{r, s}^{(n)} d r \\
& \overleftarrow{\Psi}_{t, s}=\overleftarrow{U}_{t, s}+\int_{s}^{t} \overleftarrow{U}_{t, r} Q(r) \overleftarrow{\Psi}_{r, s} d r
\end{aligned}
$$

Then for each $s \in \mathcal{I}$ here exists a limit $\operatorname{sim}_{n \rightarrow+\infty} \overleftarrow{\Psi}_{t, s}^{(n)}=\overleftarrow{\Psi}_{t, s}$ uniformly with respect to $t \in[s, T]$.

Proof. Let $\left\|\overleftarrow{U}_{t, s}\right\| \leq M_{U}$. By the uniform boundedness principle, the inequalities $\left\|Q_{n}(t)\right\|,\|Q(t)\| \leq M_{Q}$ hold with some constant $M_{Q}$. The definition of evolution families implies the relation

$$
\begin{aligned}
\overleftarrow{\Psi}_{t, s}^{(n)}-\overleftarrow{\Psi}_{t, s}= & \int_{s}^{t} \overleftarrow{U}_{t, r}\left[Q_{n}(r) \overleftarrow{\Psi}_{r, s}^{(n)}-Q(r) \overleftarrow{\Psi}_{r, s}\right] d r= \\
& \int_{s}^{t} \overleftarrow{U}_{t, r}\left[Q_{n}(r)-Q(r)\right] \overleftarrow{\Psi}_{r, s} d r+\int_{s}^{r} \overleftarrow{U}_{t, r} Q_{n}(r)\left[\overleftarrow{\Psi}_{r, s}^{(n)}-\overleftarrow{\Psi}_{r, s}\right] d r
\end{aligned}
$$

Hence for an arbitrary $x \in Y$ we obtain

$$
\begin{aligned}
\left\|\left(\overleftarrow{\Psi}_{t, s}^{(n)}-\overleftarrow{\Psi}_{t, s}\right) x\right\| \leq M_{U} \int_{s}^{t}\left\|\left[Q_{n}(r)-Q(r)\right] \overleftarrow{\Psi}_{r, s} x\right\| d r+ \\
\quad \int_{s}^{t} M_{U} M_{Q}\left\|\left(\overleftarrow{\Psi}_{r, s}^{(n)}-\overleftarrow{\Psi}_{r, s}\right) x\right\| d r
\end{aligned}
$$

Since the first integral term is monotone nondecreasing with respect to $t$, it follows from the Gronwall inequality that

$$
\left\|\left(\overleftarrow{\Psi}_{t, s}^{(n)}-\overleftarrow{\Psi}_{t, s}\right) x\right\| \leq M_{U} \exp \left(M_{U} M_{Q}(t-s)\right) \int_{s}^{t}\left\|\left[Q_{n}(r)-Q(r)\right] \overleftarrow{\Psi}_{r, s} x\right\| d r
$$


This, together with the uniform strong convergence of the sequence $Q_{n}$ to $Q$ and the uniform boundedness of the strongly continuous family $\overleftarrow{\Psi}_{t, s}$, implies that $Q_{n}(r) \overleftarrow{\Psi}_{r, s}$ strongly converges to $Q(r) \overleftarrow{\Psi}_{r, s}$ uniformly with respect to $r \in[s, T]$. The proof of the proposition is complete.

Corollary 1. Let $\vec{U}_{t, s}$ be a uniformly bounded backward evolution family in $\mathcal{L}(Y)$, and let a sequence $\left\{Q_{n}\right\}_{n=1}^{+\infty}$ converge to $Q$ in the space $C_{s}(\mathcal{I} ; \mathcal{L}(Y))$. Further, let strongly continuous backward evolution families $\vec{\Psi}_{t, s}^{(n)}$ and $\vec{\Psi}_{t, s}$ be solutions of the equations $(0 \leq t \leq s \leq T)$

$$
\begin{aligned}
\vec{\Psi}_{t, s}^{(n)} & =\vec{U}_{t, s}+\int_{t}^{s} \vec{U}_{t, r} Q_{n}(r) \vec{\Psi}_{r, s}^{(n)} d r \\
\vec{\Psi}_{t, s} & =\vec{U}_{t, s}+\int_{t}^{s} \vec{U}_{t, r} Q(r) \vec{\Psi}_{r, s} d r
\end{aligned}
$$

Then for each $t \in \mathcal{I}$ there exists a limit $\operatorname{sim}_{n \rightarrow+\infty} \vec{\Psi}_{t, s}^{(n)}=\vec{\Psi}_{t, s}$ uniformly with respect $s \in[t, T]$.

\section{Representation of the solution of the Riccati equation}

2.1. We will need the following results on the form of solutions of integral equations. Just as before, let $X_{1}$ and $X_{2}$ be be Banach spaces, and let the following conditions be satisfied:

1. $\overleftarrow{U}_{t, s}$ is a strongly continuous uniformly bounded forward evolution family in $\mathcal{L}\left(X_{1}\right)$;

2. $\vec{U}_{t, s}$ is a strongly continuous uniformly bounded backward evolution family in $\mathcal{L}\left(X_{2}\right)$;

3. The operator functions $Q_{12}, Q_{1}$ and $Q_{2}$ satisfy the inclusions

$$
Q_{12} \in C_{s}\left(\mathcal{I} ; \mathcal{L}\left(X_{1}, X_{2}\right)\right), \quad Q_{1} \in C_{s}\left(\mathcal{I} ; \mathcal{L}\left(X_{1}\right)\right), \quad Q_{2} \in C_{s}\left(\mathcal{I} ; \mathcal{L}\left(X_{2}\right)\right) .
$$


Proposition 2. Let conditions 1, 2 and 3 be satisfied, and let a strongly continuous uniformly bounded forward evolution family $\left\{\overleftarrow{\Omega}_{t, s}\right\}_{0 \leq s \leq t \leq T}$ in $\mathcal{L}\left(X_{1}\right)$ be the unique solution of the equation

$$
\overleftarrow{\Omega}_{t, s}=\overleftarrow{U}_{t, s}-\int_{s}^{t} \overleftarrow{\Omega}_{t, r} Q_{1}(r) \overleftarrow{U}_{r, s} d r, \quad 0 \leq s \leq t \leq T
$$

Then for an arbitrary $G \in \mathcal{L}\left(X_{1}, X_{2}\right)$ the equation

$$
P(t)=\vec{U}_{t, T} G \overleftarrow{U}_{T, t}+\int_{t}^{T} \vec{U}_{t, r}\left[Q_{12}(r)-P(r) Q_{1}(r)\right] \overleftarrow{U}_{r, t} d r
$$

for an operator function $P \in C_{s}\left(\mathcal{I} ; \mathcal{L}\left(X_{1}, X_{2}\right)\right)$ has the unique solution

$$
P(t)=\vec{U}_{t, T} G \overleftarrow{\Omega}_{T, t}+\int_{t}^{T} \vec{U}_{t, r} Q_{12}(r) \overleftarrow{\Omega}_{r, t} d r, \quad t \in \mathcal{I}
$$

Proof. Let us substitute the operator function (3) into the right-hand side of Eq. (2). Taking into account the semigroup property of $\vec{U}_{t, s}$, and the definition of the family $\overleftarrow{\Omega}_{t, s}$ and changing the order of integration, we obtain

$$
\begin{gathered}
\vec{U}_{t, T} G \overleftarrow{U}_{T, t}+\int_{t}^{T} \vec{U}_{t, r}\left[Q_{12}(r)-P(r) Q_{1}(r)\right] \overleftarrow{U}_{r, t} d r=\vec{U}_{t, T} G \overleftarrow{U}_{T, t}+ \\
\int_{t}^{T} \vec{U}_{t, r}\left[Q_{12}(r)-\left[\vec{U}_{r, T} G \overleftarrow{\Omega}_{T, r}+\int_{r}^{T} \vec{U}_{r, s} Q_{12}(s) \overleftarrow{\Omega}_{s, r} d s\right] Q_{1}(r)\right] \overleftarrow{U}_{r, t} d r= \\
\vec{U}_{t, T} G\left[\overleftarrow{U}_{T, t}-\int_{t}^{T} \overleftarrow{\Omega}_{T, r} Q_{1}(r) \overleftarrow{U}_{T, r} d r\right]+\int_{t}^{T} \vec{U}_{t, r} Q_{12}(r) \overleftarrow{U}_{r, t} d r- \\
\int_{t}^{T} \vec{U}_{t, s} Q_{12}(s) \overleftarrow{U}_{s, t} d s-\int_{t}^{T}\left[\int_{t}^{s} \vec{U}_{t, s} Q_{12}(s) \overleftarrow{\Omega}_{s, r} Q_{1}(r) \overleftarrow{\Omega}_{s, r} \overleftarrow{Q}_{1}(r) \overleftarrow{U}_{r, t} d s\right] d r=\vec{U}_{t, T} G \overleftarrow{\Omega}_{T, t}+ \\
\vec{U}_{t, T} G \overleftarrow{\Omega}_{T, t}+\int_{t}^{T} \vec{U}_{t, s} Q_{12}(s)\left[\overleftarrow{U}_{s, t}-\int_{t}^{s} \overleftarrow{\Omega}_{s, r} Q_{1}(r) \overleftarrow{U}_{r, t} d r\right] d s= \\
\vec{U}_{t, T} G \overleftarrow{\Omega}_{T, t}+\int_{t}^{T} \vec{U}_{t, s} Q_{12}(s) \overleftarrow{\Omega}_{s, t} d s=P(t)
\end{gathered}
$$

Thus, the operator function (3) satisfies Eq. (2). 
The uniqueness of the solution of Eq. (2) follows from the fact that, for sufficiently small $\delta>0$ the mapping $F$ acting by the rule

$$
F(P)(t)=\vec{U}_{t, \tau} G \overleftarrow{U}_{\tau, t}+\int_{t}^{\tau} \vec{U}_{t, r}\left[Q_{12}(r)-P(r) Q_{1}(r)\right] \overleftarrow{U}_{r, t} d r
$$

is a contraction on the space $C_{u}\left([\tau-\delta, \tau] ; \mathcal{L}\left(X_{1}, X_{2}\right)\right)$ for all $\delta \leq \tau \leq T$. The proof of the proposition is complete.

In a similar way, one can prove the following proposition.

Proposition 3. Let conditions 1, 2 and 3 be satisfied, and let a strongly continuous uniformly bounded backward evolution family $\vec{\Omega}_{t, s}$ in $\mathcal{L}\left(X_{2}\right)$ be the unique solution of the equation

$$
\vec{\Omega}_{t, s}=\vec{U}_{t, s}-\int_{t}^{s} \vec{U}_{t, r} Q_{2}(r) \vec{\Omega}_{r, s} d r, \quad 0 \leq t \leq s \leq T .
$$

Then for an arbitrary $G \in \mathcal{L}\left(X_{1}, X_{2}\right)$ the equation

$$
P(t)=\vec{U}_{t, T} G \overleftarrow{U}_{T, t}+\int_{t}^{T} \vec{U}_{t, r}\left[Q_{12}(r)-Q_{2}(r) P(r)\right] \overleftarrow{U}_{r, t} d r
$$

for an operator function $P \in C_{s}\left(\mathcal{I} ; \mathcal{L}\left(X_{1}, X_{2}\right)\right)$ has the unique solution

$$
P(t)=\vec{\Omega}_{t, T} G \overleftarrow{U}_{T, t}+\int_{t}^{T} \vec{\Omega}_{t, r} Q_{12}(r) \overleftarrow{U}_{r, t} d r
$$

Proposition 4. Let conditions 1, 2 and 3 be satisfied, and let strongly continuous uniformly bounded (forward and backward) evolution families $\overleftarrow{\Omega}_{t, s}$ and $\vec{\Omega}_{t, s}$ be defined in the same way as in Propositions 2 and 3, respectively. Then for an arbitrary $G \in \mathcal{L}\left(X_{1}, X_{2}\right)$ the equation

$$
P(t)=\vec{U}_{t, T} G \overleftarrow{U}_{T, t}+\int_{t}^{T} \vec{U}_{t, r}\left[Q_{12}(r)-P(r) Q_{1}(r)-Q_{2}(r) P(r)\right] \overleftarrow{U}_{r, t} d r
$$

for an operator function $P \in C_{s}\left(\mathcal{I} ; \mathcal{L}\left(X_{1}, X_{2}\right)\right)$ has the unique solution

$$
P(t)=\vec{\Omega}_{t, T} G \overleftarrow{\Omega}_{T, t}+\int_{t}^{T} \vec{\Omega}_{t, r} Q_{12}(r) \overleftarrow{\Omega}_{r, t} d r
$$


Proof. Set $\tilde{Q}_{12}(t)=Q_{12}(t)-P(t) Q_{1}(t)$. Then Eq. (6) can be written in the form

$$
P(t)=\vec{U}_{t, T} G \overleftarrow{U}_{T, t}+\int_{t}^{T} \vec{U}_{t, r}\left[\tilde{Q}_{12}(r)-Q_{2}(r) P(r)\right] \overleftarrow{U}_{r, t} d r
$$

By Proposition 3, its solution has the form

$$
P(t)=\vec{\Omega}_{t, T} G \overleftarrow{U}_{T, t}+\int_{t}^{T} \vec{\Omega}_{t, r} \tilde{Q}_{12}(r) \overleftarrow{U}_{r, t} d r
$$

i.e., the operator function $P$ satisfies the equation

$$
P(t)=\vec{\Omega}_{t, T} G \overleftarrow{U}_{T, t}+\int_{t}^{T} \vec{\Omega}_{t, r}\left(Q_{12}(r)-P(r) Q_{1}(r)\right) \overleftarrow{U}_{r, t} d r
$$

By Proposition 2 the unique solution of this equation (and hence of Eq. (6)) has the form (7)

2.2. Let us prove some results on the representation of the solution of the Riccati integral equation (1).

Proposition 5. Let $P \in C_{s}\left(\mathcal{I} ; \mathcal{L}\left(X_{1}, X_{2}\right)\right)$, and let an evolution family $\overleftarrow{\Psi}_{t, s}$ in $\mathcal{L}\left(X_{1}\right)$ be a solution of the equation

$$
\overleftarrow{\Psi}_{t, s}=\overleftarrow{U}_{t, s}-\int_{s}^{t} \overleftarrow{\Psi}_{t, r} B(r) P(r) \overleftarrow{U}_{r, s} d r \quad 0 \leq s \leq t \leq T
$$

The operator function $P$ is a solution of the Riccati integral equation (1) if and only if

$$
P(t)=\vec{U}_{t, T} G \overleftarrow{\Psi}_{T, t}+\int_{t}^{T} \vec{U}_{t, r} C(r) \overleftarrow{\Psi}_{r, t} d r
$$

for each $t \in \mathcal{I}$.

Proof. The proof readily follows from Proposition 2, where one must set $Q_{1}(t)=B(t) P(t)$ and $\overleftarrow{\Omega}_{t, s}=\overleftarrow{\Psi}_{t, s}$

Proposition 6. Let $P \in C_{s}\left(\mathcal{I} ; \mathcal{L}\left(X_{1}, X_{2}\right)\right)$, let a forward evolution famuily $\overleftarrow{\Psi}_{t, s}$ in $\mathcal{L}\left(X_{1}\right)$ be a solution of Eq. (8), and let a backward evolution family $\vec{\Psi}_{s, t}$ in $\mathcal{L}\left(X_{2}\right)$ be a solution of the equation

$$
\vec{\Psi}_{t, s}=\vec{U}_{t, s}-\int_{t}^{s} \vec{U}_{t, r} P(r) B(r) \vec{\Psi}_{r, s} d r \quad 0 \leq t \leq s \leq T
$$


The operator function $P$ is a solution of the Riccati equation (1) if and only if the relation

$$
P(t)=\vec{\Psi}_{t, T} G \overleftarrow{\Psi}_{T, t}+\int_{t}^{T} \vec{\Psi}_{t, r}(C(r)+P(r) B(r) P(r)) \overleftarrow{\Psi}_{r, t} d r
$$

holds for each $t \in \mathcal{I}$.

Proof. The proof readily follows from Proposition 4, where one must set $\vec{\Omega}_{t, s}=\vec{\Psi}_{t, s}, \overleftarrow{\Omega}_{t, s}=\overleftarrow{\Psi}_{t, s}, Q_{1}(t)=B(t) P(t), Q_{2}(t)=P(t) B(t)$ and $Q_{12}(t)=$ $C(t)+P(t) B(t) P(t)$.

2.3. To prove the uniqueness of the solution of the Riccati integral equation, we need a generalization of the following result [1, Sec. IV, Lemma $2.2]$

Proposition 7. Let

$$
Q_{0} \in C_{u}\left([a, b] ; \mathcal{L}\left(X_{1}, X_{2}\right)\right), \quad B \in C_{u}\left([a, b] ; \mathcal{L}\left(X_{2}, X_{1}\right)\right)
$$

and let operator functions $\left\{Q_{s, t}^{(1,2)}\right\}_{a \leq t \leq s \leq b}$ ranging in $\mathcal{L}\left(X_{1,2}\right)$ be strongly continuous separately in $t$ and $s$ and uniformly bounded, $\left\|Q_{s, t}^{(1,2)}\right\|_{\mathcal{L}\left(X_{1,2}\right)} \leq M_{1,2}$. Further, let $\rho$ be a number satisfying the inequalities

$$
\left\|Q_{0}\right\|_{u}+\rho^{2}(b-a) M_{1} M_{2}\|B\|_{u} \leq \rho, \quad 2 \rho(b-a) M_{1} M_{2}\|B\|_{u}<1
$$

Then the mapping $\Gamma$ acting on the space $C_{u}\left([a, b] ; \mathcal{L}\left(X_{1}, X_{2}\right)\right)$ by the rule

$$
\Gamma(P)(t)=Q_{0}(t)-\int_{t}^{b} Q_{r, t}^{(2)} P(r) B(r) P(r) Q_{r, t}^{(1)} d r
$$

is a contraction on the ball

$$
B_{\rho}=\left\{P \in C_{u}\left([a, b] ; \mathcal{L}\left(X_{1}, X_{2}\right)\right):\|P\|_{u} \leq \rho\right\} .
$$

Proof. Let us show that $\Gamma\left(B_{\rho}\right) \subseteq B_{\rho}$. Let $P \in B_{\rho}$. For each $x \in X_{1}$, we have

$$
\left\|Q_{r, t}^{(2)} P(r) B(r) P(r) Q_{r, t}^{(1)} x\right\|_{X_{2}} \leq \rho^{2} M_{1} M_{2}\|B\|_{u}\|x\|_{X_{1}} .
$$


and hence

$$
\begin{gathered}
\|\Gamma(P)(t) x\|_{X_{2}}=\left\|Q_{0}(t)-\int_{t}^{b} Q_{r, t}^{(2)} P(r) B(r) P(r) Q_{r, t}^{(1)} x d r\right\|_{X_{2}} \leq \\
\left\|Q_{0}(t) x\right\|_{X_{2}}+\int_{t}^{b}\left\|Q_{r, t}^{(2)} P(r) B(r) P(r) Q_{r, t}^{(1)} x\right\|_{X_{2}} d r \leq \\
\left(\left\|Q_{0}\right\|_{u}+\rho^{2} M_{1} M_{2}(b-t)\|B\|_{u}\right)\|x\|_{X_{1}} .
\end{gathered}
$$

Since $x$ and $t \in[a, b]$ are arbitrary, we take into account the first inequality in (12) and obtain

$$
\|\Gamma(P)\|_{u} \leq\left\|Q_{0}\right\|_{u}+\rho^{2}(b-a) M_{1} M_{2}\|B\|_{u} \leq \rho,
$$

i.e., $\Gamma(P) \in B_{\rho}$. Further, let $P_{1}, P_{2} \in B_{\rho}$. For each $x \in X_{1}$,

$$
\begin{aligned}
& \left\|Q_{r, t}^{(2)}\left\{P_{2}(r) B(r) P_{2}(r)-P_{1}(r) B(r) P_{1}(r)\right\} Q_{r, t}^{(1)} x\right\|_{X_{2}}= \\
& M_{1} M_{2}\left\|\left(P_{2}(r)-P_{1}(r)\right) B(r) P_{2}(r)+P_{1}(r) B(r)\left(P_{2}(r)-P_{1}(r)\right)\right\|\|x\|_{X_{1}} \leq \\
& M_{1} M_{2}\left\|P_{2}(r)-P_{1}(r)\right\|\|B(r)\|\left\{\left\|P_{1}(r)\right\|+\left\|P_{2}(r)\right\|\right\}\|x\|_{X_{1}} \leq \\
& 2 \rho M_{1} M_{2}\left\|P_{2}-P_{1}\right\|_{u}\|B\|_{u}\|x\|_{X_{1}} .
\end{aligned}
$$

Then

$$
\begin{aligned}
& \left\|\Gamma\left(P_{1}\right)(t) x-\Gamma\left(P_{2}\right)(t) x\right\|_{X_{2}}= \\
& \qquad \int_{t}^{b} Q_{r, t}^{(2)}\left\{P_{2}(r) B(r) P_{2}(r)-P_{1}(r) B(r) P_{1}(r)\right\} Q_{r, t}^{(1)} x d r \|_{X_{2}} \leq \\
& \int_{t}^{b}\left\|Q_{r, t}^{(2)}\left\{P_{2}(r) B(r) P_{2}(r)-P_{1}(r) B(r) P_{1}(r)\right\} Q_{r, t}^{(1)} x\right\|_{X_{2}} d r \leq \\
& 2 \rho(b-t) M_{1} M_{2}\left\|P_{2}-P_{1}\right\|_{u}\|B\|_{u}\|x\|_{X_{1}} .
\end{aligned}
$$

or, finally,

$$
\left\|\Gamma\left(P_{1}\right)-\Gamma\left(P_{2}\right)\right\|_{u} \leq\left\{2 \rho(b-a) M_{1} M_{2}\|B\|_{u}\right\}\left\|P_{1}-P_{2}\right\|_{u} .
$$

Since $2 \rho(b-a) M_{1} M_{2}\|B\|_{u}<1$ by the second inequality in (12) it follows that the mapping $\Gamma$ in the space $C_{u}\left([a, b] ; \mathcal{L}\left(X_{1}, X_{2}\right)\right)$ is a contraction mapping of the ball $B_{\rho}$ into itself. The proof of the proposition is complete. 
Corollary 2. Under the assumptions of Proposition \%, the equation

$$
P(t)=Q_{0}(t)-\int_{t}^{b} Q_{r, t}^{(2)} P(r) B(r) P(r) Q_{r, t}^{(1)} d r
$$

has a unique solution $P \in C_{u}\left([a, b] ; \mathcal{L}\left(X_{1}, X_{2}\right)\right)$ with $\|P\|_{u} \leq \rho$.

Proposition 8. Let numbers $r_{B}, r_{C}$ and $r_{G}$ satisfy the inequality

$$
4(b-a) M_{1}^{2} M_{2}^{2}\left(r_{G}+(b-a) r_{C}\right) r_{B}<1
$$

and let operator functions $Q_{s, t}^{(1,2)}$ satisfy the assumptions of Proposition $\%$ Then the equation

$$
P(t)=Q_{b, t}^{(2)} G Q_{b, t}^{(1)}+\int_{t}^{b} Q_{r, t}^{(2)}\{C(r)-P(r) B(r) P(r)\} Q_{r, t}^{(1)} d r
$$

has a unique solution $P \in C_{u}\left([a, b] ; \mathcal{L}\left(X_{1}, X_{2}\right)\right)$ for arbitrary $G \in \mathcal{L}\left(X_{1}, X_{2}\right)$, $C \in C_{u}\left([a, b] ; \mathcal{L}\left(X_{1}, X_{2}\right)\right)$ and $B \in C_{u}\left([a, b] ; \mathcal{L}\left(X_{2}, X_{1}\right)\right)$ such that

$$
\|G\|_{\mathcal{L}\left(X_{1}, X_{2}\right)} \leq r_{G} \quad\|C\|_{u} \leq r_{C} \quad\|B\|_{u} \leq r_{B},
$$

and one has $\|P\|_{u} \leq 2 M_{1} M_{2}\left(r_{G}+(b-a) r_{C}\right)$.

Proof. Set

$$
Q_{0}(t)=Q_{b, t}^{(2)} G Q_{b, t}^{(1)}+\int_{t}^{b} Q_{r, t}^{(2)} C(r) Q_{r, t}^{(1)} d r
$$

Then Eq. (13) can be written in the form $P=\Gamma(P)$, where the mapping $\Gamma(\cdot)$ is defined in Proposition 7. Let $\rho=2 M_{1} M_{2}\left(r_{G}+(b-a) r_{C}\right)$. By virtue of the assumptions in the proposition to be proved, we have

$$
2 \rho(b-a) M_{1} M_{2}\|B\|_{u} \leq 4 M_{1}^{2} M_{2}^{2}(b-a)\left(r_{G}+(b-a) r_{C}\right) r_{B}<1 .
$$

Further, since

$$
\left\|Q_{0}\right\|_{u} \leq M_{1} M_{2}\left(\|G\|_{\mathcal{L}\left(X_{1}, X_{2}\right)}+(b-a)\|C\|_{u}\right) \leq M_{1} M_{2}\left(r_{C}+(b-a) r_{C}\right),
$$

we have

$$
\begin{aligned}
& \left\|Q_{0}\right\|_{u}+\rho^{2}(b-a) M_{1} M_{1}\|B\|_{u} \leq \\
& M_{1} M_{2}\left(\|G\|_{\mathcal{L}\left(X_{1}, X_{2}\right)}+(b-a)\|C\|_{u}\right)+\rho^{2}(b-a) M_{1} M_{1}\|B\|_{u}< \\
& M_{1} M_{2}\left(r_{G}+(b-a) r_{C}\right)+\rho / 2=\rho .
\end{aligned}
$$

Hence inequalities (12) are satisfied for the number $\rho$. Now the unique solvability of Eq. (13) follows from Proposition 7 and Corollary 2. The proof of the proposition is complete. 


\section{Main result}

Let $X$ be a reflexive Banach space. The duality between the spaces $X$ and $X^{*}$ will be denoted by $\langle y, x\rangle$, where $y \in X^{*}$ and $x \in X$.

Let $A_{1} \in \mathcal{L}\left(X, X^{*}\right)$. Then the adjoint operator $A_{1}^{*} \in \mathcal{L}\left(X^{* *}, X^{*}\right)$. Using the canonical isomorphism between the spaces $X^{* *}$ and $X$ we can treat the adjoint operator as $A_{1}^{*} \in \mathcal{L}\left(X, X^{*}\right)$. A straightforward verification shows that

$$
\left\langle A_{1} x_{1}, x_{2}\right\rangle=\overline{\left\langle A_{1}^{*} x_{2}, x_{1}\right\rangle} \quad \forall x_{1}, x_{2} \in X .
$$

Definition 5. An operator $A_{1} \in \mathcal{L}\left(X, X^{*}\right)$ is said to be self-adjoint if $A_{1}=$ $A_{1}^{*}$. This is equivalent to the condition that $\left\langle A_{1} x, x\right\rangle \in \mathbb{R}$ for all $x \in X$.

We say that a self-adjoint operator $A_{1} \in \mathcal{L}\left(X, X^{*}\right)$ is nonnegative and write $A_{1} \geq 0$ if $\left\langle A_{1} x, x\right\rangle \geq 0$ for all $x \in X$.

In a similar way, if $A_{2} \in \mathcal{L}\left(X^{*}, X\right)$, then, identifying the spaces $X^{* *}$ and $X$, we assume that $A_{2}^{*} \in \mathcal{L}\left(X^{*}, X\right)$; a straightforward verification shows that

$$
\left\langle x_{1}, A_{2} x_{2}\right\rangle=\overline{\left\langle x_{2}, A_{2}^{*} x_{1}\right\rangle} \quad \forall x_{1}, x_{2} \in X^{*} .
$$

Definition 6. An operator $A_{2} \in \mathcal{L}\left(X^{*}, X\right)$ is said to be elf-adjoint, if $A_{2}=$ $A_{2}^{*}$. This is equivalent to the condition that $\left\langle x, A_{2} x\right\rangle \in \mathbb{R}$ for all $s x \in X^{*}$.

We say that a self-adjoint operator $A_{2} \in \mathcal{L}\left(X^{*}, X\right)$ is nonnegative and write $A_{2} \geq 0$ if $\left\langle x, A_{2} x\right\rangle \geq 0$ for all $x \in X^{*}$.

Let us state the main result about the unique solvability of the Riccati integral equation.

Theorem 2. Let $X$ be a reflexive Banach space, and let the following conditions be satisfied:

1. $\left\{\overleftarrow{U}_{t, s}\right\}_{0 \leq s \leq t \leq T}$ is a strongly continuous uniformly bounded forward evolution family in $\mathcal{L}(X)$;

2. $\vec{U}_{t, s}=\left(\overleftarrow{U}_{s, t}\right)^{*}$ is a backward evolution family. (Since $X$ is s reflexive, it follows that this family is strongly continuous and uniformly bounded in $\left.\mathcal{L}\left(X^{*}\right)\right)$;

3. The operator functions $C$ and $B$ satisfy the inclusions

$$
C \in C_{s}\left(\mathcal{I} ; \mathcal{L}\left(X, X^{*}\right)\right) \quad \text { and } \quad B \in C_{s}\left(\mathcal{I} ; \mathcal{L}\left(X^{*}, X\right)\right)
$$




$$
\text { 4. } C(t)=C^{*}(t) \geq 0 \text { and } B(t)=B^{*}(t) \geq 0 \text { for all } t \in \mathcal{I} \text {. }
$$

Then for an arbitrary self-adjoint nonnegative operator $G \in \mathcal{L}\left(X, X^{*}\right)$ the Riccati integral equation (1) has a unique solution $P \in C_{s}\left(\mathcal{I} ; \mathcal{L}\left(X, X^{*}\right)\right)$ and $P(t)=P^{*}(t) \geq 0$ for all $t \in \mathcal{I}$.

Proof. Following [1, 2, 5], consider the sequence

$$
\left\{P_{n}\right\}_{n=0}^{+\infty} \subset C_{s}\left(\mathcal{I} ; \mathcal{L}\left(X, X^{*}\right)\right)
$$

of operator functions defined recursively as follows. Set $P_{0}(t) \equiv 0$ and define $P_{n+1}(t)$ as the solution of the equation

$$
\begin{aligned}
& P_{n+1}(t)=\vec{U}_{t, T} G \overleftarrow{U}_{T, t}+\int_{t}^{T} \vec{U}_{t, r}\{C(r)+ \\
& \left.\quad P_{n}(r) B(r) P_{n}(r)-P_{n+1}(r) B(r) P_{n}(r)-P_{n}(r) B(r) P_{n+1}(r)\right\} \overleftarrow{U}_{r, t} d r
\end{aligned}
$$

for each $n \geq 0$. By $\overleftarrow{\Psi}_{t, s}^{(n)} \in \mathcal{L}(X)$ and $\vec{\Psi}_{s, t}^{(n)} \in \mathcal{L}\left(X^{*}\right)$ we denote the solutions of Eqs. (8) and (10), respectively, with $P=P_{n}$. By Proposition 4, the solution of Eq. (14) has the form

$$
P_{n+1}(t)=\vec{\Psi}_{t, T}^{(n)} G \overleftarrow{\Psi}_{T, t}^{(n)}+\int_{t}^{T} \vec{\Psi}_{t, r}^{(n)}\left\{C(r)+P_{n}(r) B(r) P_{n}(r)\right\} \overleftarrow{\Psi}_{r, t}^{(n)} d r
$$

If $P_{n}^{*}(t)=P_{n}(t)$ for all $t \in \mathcal{I}$, then it follows from Eqs. (8) and (10), the selfadjointness of the operator function $B(t)$ and the condition $\vec{U}_{t, s}=\left(\overleftarrow{U}_{s, t}\right)^{*}$ that $\vec{\Psi}_{t, s}^{(n)}=\left(\overleftarrow{\Psi}_{s, t}^{(n)}\right)^{*}$

In view of this equality, it readily follows from Eq. (15) that the selfadjointness of the operator function $P_{n}$ implies the self-adjointness of the operator function $P_{n+1}$. Since $P_{0}=0$, we see that $\left\{P_{n}\right\}_{n=0}^{+\infty}$ is a sequence of self-adjoint operator functions. Further, a straightforward verification for Eq. (15) shows that the nonnegativity of the operators $(t \in \mathcal{I})$

$$
G, C(t), B(t) \geq 0
$$

implies the nonnegativity of the operator function $P_{n+1}$

Let us show that $P_{n+1}(t) \leq P_{n}(t)$ (i.e. $\left.P_{n}(t)-P_{n+1}(t) \geq 0\right)$ for all $t \in[0, T]$ and $n \in \mathbb{N}$. Let $Q_{n+1}=P_{n+1}-P_{n}$. Let us subtract Eq. (14) written for 
$P_{n}$ from from the same equation for $P_{n+1}$. After obvious transformations, we find that the operator function $Q_{n+1}$ satisfies the equation

$$
\begin{aligned}
Q_{n+1}(t)=-\int_{t}^{T} \vec{U}_{t, r}\left\{Q_{n}(r) B(r) Q_{n}(r)+\right. & \\
\left.Q_{n+1}(r) B(r) P_{n}(r)+P_{n}(r) B(r) Q_{n+1}(r)\right\} & \overleftarrow{U}_{r, t} d r
\end{aligned}
$$

By Proposition 4, the unique solution of this equation has the form

$$
Q_{n+1}(t)=-\int_{t}^{T} \vec{\Psi}_{t, r}^{(n)} Q_{n}(r) B(r) Q_{n}(r) \overleftarrow{\Psi}_{r, t}^{(n)} d r
$$

Since $Q_{n}^{*}(t)=Q_{n}(t)$ and $B(t) \geq 0$, we have $Q_{n+1}(t)=P_{n+1}(t)-P_{n}(t) \leq 0$ for all $t \in \mathcal{I}$.

Thus, $\left\{P_{n}(t)\right\}_{n=1}^{+\infty}$ is a monotone nonincreasing sequence of nonnegative self-adjoint operators in $\mathcal{L}\left(X, X^{*}\right)$ for each $t \in \mathcal{I}$. Then [11, Th. 4] for each $t \in \mathcal{I}$ there exists a strong limit

$$
\operatorname{sim}_{n \rightarrow+\infty} P_{n}(t)=P(t) \in \mathcal{L}\left(X, X^{*}\right),
$$

and $P^{*}(t)=P(t) \geq 0$. Moreover, since [11, Th. 4]

$$
\left\|P_{n}(t)\right\|_{\mathcal{L}\left(X, X^{*}\right)}=\sup _{x \in X,\|x\|=1}\left\langle P_{n}(t) x, x\right\rangle,
$$

we see that the numerical sequence $\left\|P_{n}(t)\right\|_{\mathcal{L}\left(X, X^{*}\right)}$ is monotone decreasing for each $t \in \mathcal{I}$.

Since $\left\{P_{n}\right\}_{n=0}^{+\infty}$ are uniformly bounded, then there exist strong limits

$$
\begin{array}{r}
\operatorname{sim}_{n \rightarrow+\infty} \vec{U}_{t, r} P_{n}(r) B(r) P_{n}(r) \overleftarrow{U}_{r, t}=\operatorname{sim}_{n \rightarrow+\infty} \vec{U}_{t, r} P_{n+1}(r) B(r) P_{n}(r) \overleftarrow{U}_{r, t}= \\
\operatorname{sim}_{n \rightarrow+\infty} \vec{U}_{t, r} P_{n}(r) B(r) P_{n+1}(r) \overleftarrow{U}_{r, t}=\vec{U}_{t, r} P(r) B(r) P(r) \overleftarrow{U}_{r, t}
\end{array}
$$

for each $0 \leq t \leq r \leq T$.

Let us fix an $x \in X$ and write $(t \leq r)$

$$
\begin{gathered}
f_{n}(t)=\left\langle P_{n}(t) x, x\right\rangle, \quad f(t)=\langle P(t) x, x\rangle \\
g(t, r)=-\left\langle\vec{U}_{t, r} P(r) B(r) P(r) \overleftarrow{U}_{r, t} x, x\right\rangle \\
h(t)=\left\langle\vec{U}_{t, T} G \overleftarrow{U}_{T, t} x\right\rangle+\int_{t}^{T}\left\langle\vec{U}_{t, r} C(r) \overleftarrow{U}_{r, t} x, x\right\rangle d r
\end{gathered}
$$


and

$$
\begin{aligned}
g_{n}(t, r)=\left\langle\vec{U}_{t, r} P_{n}(r) B(r) P_{n}(r) \overleftarrow{U}_{r, t} x, x\right\rangle- \\
\\
\left.\left\langle\vec{U}_{t, r}\left\{P_{n+1}(r) B(r) P_{n}(r)+P_{n}(r) B(r) P_{n+1}(r)\right]\right\} \overleftarrow{U}_{r, t} x, x\right\rangle
\end{aligned}
$$

By construction,

- $f_{n} \in C(\mathcal{I}), f_{n}(t) \geq 0, f_{n+1}(t) \leq f_{n}(t)$ and the sequence $f_{n}(t)$ converges to $f(t)$ pointwise on $\mathcal{I}$;

- $g_{n}(t, \cdot) \in C([t, T])$ are uniformly bounded and the sequence $g_{n}(t, \cdot)$ converges to $g(t, \cdot)$ pointwise on $[t, T]$ for each $t \in \mathcal{I}$;

- $g_{n}(\cdot, r), g(\cdot, r) \in C([0, r])$ for each $r \in \mathcal{I}$;

- $h \in C(\mathcal{I})$ and Eq. (14) implies the equality $(t \in \mathcal{I})$

$$
f_{n}(t)=h(t)+\int_{t}^{T} g_{n}(t, r) d r
$$

Thus $f$ and $g(t, \cdot)$ are bounded and measurable. Passing to the limit as $n \rightarrow+\infty$ in $(16)$ we obtain $(t \in \mathcal{I})$

$$
f(t)=h(t)+\int_{t}^{T} g(t, r) d r .
$$

Since $g(\cdot, r)$ is continuous for each $r \in \mathcal{I}$ and uniformely bounded, then $\int_{t}^{T} g(t, r) d r \in C(\mathcal{I})$. Thus $f \in C(\mathcal{I})$.

By virtue of Dini's theorem the functional sequence $\left\{f_{n}\right\}_{n=1}^{+\infty}$ converges to $f$ uniformly on $\mathcal{I}$. Further, for any $n, m \in \mathbb{N}$ the inequality $P_{n+m}(t) \leq P_{n}(t)$ implies the estimate [11, Th. 4]

$$
\begin{aligned}
& \left\|P_{n}(t) x-P_{n+m}(t) x\right\|_{X}^{2} \leq \\
& \left\|P_{n}(t)-P_{n+m}(t)\right\|_{\mathcal{L}\left(X, X^{*}\right)}\left\langle P_{n}(t) x-P_{n+m}(t) x, x\right\rangle \leq \\
& \operatorname{const}\left(f_{n}(t)-f_{n+m}(t)\right),
\end{aligned}
$$

for all $t \in \mathcal{I}$, where the constant does not depend on $t$. According to the Cauchy convergence test, we find that the uniform convergence on $\mathcal{I}$ of the sequence $\left\{f_{n}\right\}_{n=1}^{+\infty}$ implies the uniform convergence of the sequence $P_{n} x$ to 
$P x$ on $\mathcal{I}$ and the inclusion $P x \in C\left(\mathcal{I}, X^{*}\right)$. Since $x$ is arbitrary, we conclude that the sequence $\left\{P_{n}\right\}_{n=1}^{+\infty}$ converges in the space $C_{s}\left(\mathcal{I} ; \mathcal{L}\left(X, X^{*}\right)\right)$ and one has the inclusion $P \in C_{s}\left(\mathcal{I} ; \mathcal{L}\left(X, X^{*}\right)\right)$.

Let $\overleftarrow{\Psi}_{t, s} \in \mathcal{L}(X)$ and $\vec{\Psi}_{s, t} \in \mathcal{L}\left(X^{*}\right)$ be solutions of Eqs. (8) and (10), respectively. Let us show that $P$ is a solution of the Riccati integral equation (1). Since

$$
B \in C_{s}\left(\mathcal{I} ; \mathcal{L}\left(X^{*}, X\right)\right), \quad P_{n}, P \in C_{s}\left(\mathcal{I} ; \mathcal{L}\left(X, X^{*}\right)\right),
$$

it follows that the sequence $B P_{n}$ converges to $B P$ in the space $C_{s}(\mathcal{I} ; \mathcal{L}(X))$. Applying Proposition 1 with $Q_{n}=B P_{n}$, we find that there exists a limit $\operatorname{slim}_{n \rightarrow+\infty} \overleftarrow{\Psi}_{r, t}^{(n)}=\overleftarrow{\Psi}_{r, t}$ uniformly with respect to $r \in[t, T]$. It can be shown in a similar way that there exists a $\operatorname{limit}_{\operatorname{sim}} \operatorname{sim}_{n \rightarrow+\infty} \vec{\Psi}_{t, r}^{(n)}=\vec{\Psi}_{t, r}$ uniformly with respect to $r \in[t, T]$.

It follows from the uniform convergence of the sequences $P_{n}, \overleftarrow{\Psi}_{r, t}^{(n)}$ and $\vec{\Psi}_{t, r}^{(n)}$ to $P, \overleftarrow{\Psi}_{r, t}$ and $\vec{\Psi}_{t, r}$, respectively, that for each $r \in[t, T]$ the following limit exists uniformly with respect to $r \in[t, T]$

$$
\operatorname{sim}_{n \rightarrow+\infty} \vec{\Psi}_{t, r}^{(n)}\left\{C(r)+P_{n}(r) B(r) P_{n}(r)\right\} \overleftarrow{\Psi}_{r, t}^{(n)}=\vec{\Psi}_{t, r}\{C(r)+P(r) B(r) P(r)\} \overleftarrow{\Psi}_{r, t}
$$

Passing to the limit as $n \rightarrow+\infty$ in (15) we see that the operator function $P$ satisfies Eq. (11) in which the evolution families $\overleftarrow{\Psi}_{r, t}$ and $\vec{\Psi}_{t, r}$ are determined by Eqs. (8) and (10), respectively. It follows from Proposition 6 that the operator function $P \in C_{s}\left(\mathcal{I} ; \mathcal{L}\left(X, X^{*}\right)\right)$ is a solution of the Riccati integral equation (1).

Let us prove the uniqueness of the solution of this equation. Set

$$
r=\sup _{t \in \mathcal{I}}\|P(t)\|_{\mathcal{L}\left(X, X^{*}\right)} \quad r_{C}=\sup _{t \in \mathcal{I}}\|C(t)\|_{\mathcal{L}\left(X, X^{*}\right)} \quad r_{B}=\sup _{t \in \mathcal{I}}\|B(t)\|_{\mathcal{L}\left(X^{*}, X\right)}
$$

Since $P(T)=G$, we have $r \geq\|G\|_{\mathcal{L}\left(X, X^{*}\right)}$. Let the following inequality be satisfied for $\delta>0$

$$
4 \delta M_{1}^{2} M_{2}^{2}\left(r+\delta r_{C}\right) r_{B}<1 .
$$

If $Q$ is another solution of Eq. (1), then, applying Proposition 8 to the interval $[T-\delta, T]$ (setting $Q_{r, t}^{(1)}=\overleftarrow{U}_{r, t}$ and $Q_{r, t}^{(2)}=\vec{U}_{t, r}$ ), we obtain $P(t)=Q(t)$ for all $t \in[T-\delta, T]$. Further,

$$
P(t)=\vec{U}_{t, T-\delta} P(T-\delta) \overleftarrow{U}_{T-\delta, t}+\int_{t}^{T-\delta} \vec{U}_{t, r}\{C(r)-P(r) B(r) P(r)\} \overleftarrow{U}_{r, t} d r
$$


for all $0 \leq t \leq T-\delta$. Applying Proposition 8 to the interval $[T-2 \delta, T-\delta]$, we conclude that $P(t)=Q(t)$ for all $t \in[T-2 \delta, T-\delta]$. Continuing this process, we see that $P(t)=Q(t)$ for all $t \in \mathcal{I}$. This proves the uniqueness of the solution of the Riccati integral equation (1). The proof of the theorem is complete.

\section{References}

[1] Bensoussan, A., Da Prato, G., Delfour, M.C., and Mitter, S.K., Representation and Control of Infinite Dimensional Systems, Boston: Birkhaeuser, 2007.

[2] Curtain, R. and Pritchard, A.J., The infinite-dimensional Riccati equation for systems defined by evolution operators, SIAM J. Control Optim., 1976, vol. 14, no. 5, pp. 951-983.

[3] Lasiecka, I., Optimal control problems and Riccati equations for systems with unbounded controls and partially analytic generators, in Functional Analytic Methods for Evolution Equations, Berlin, 2004, pp. 313-371.

[4] Gibson, J.S., The Riccati integral equations for optimal control problems on Hilbert spaces, SIAM J. Control Optim., 1979, vol. 17, no. 4, pp. $537-565$.

[5] Pritchard, A.J. and Salamon, D., The linear quadratic control problem for infinite dimensional systems with unbounded input and output operators, SIAM J. Control Optim., 1987, vol. 25, no. 1, pp. 121-144.

[6] Lasiecka, I. and Triggiani, R., Riccati differential equations with unbounded coefficients and non-smooth terminal condition-the case of analytic semigroups, SIAM J. Math. Anal., 1992, vol. 23, no. 2, pp. 449-481.

[7] Da Prato, G. and Ichikawa, A., Riccati equations with unbounded coefficients, Ann. Mat. Pura Appl., 1985, vol. 40, no. 1, pp. 209-211.

[8] Artamonov, N.V., On the solvability of a system of forward-backward linear equations with unbounded operator coefficients, Math. Notes, 2016, vol. 100, nos. 5-6, pp. 747-750. 
[9] Yong, J., Forward-backward evolution equations and applications, 2015, arXiv: $1508.03550 \mathrm{v} 1$.

[10] Engel, K. and Nagel, R., One-parameter semigroups for linear evolution equations, in Grad. Texts in Math., Berlin: Springer-Verlag, 2000, Vol. 194.

[11] Koshkin, S., Positive semigroup and algebraic Riccati equations in Banach spaces, Positivity, 2016, vol. 20, pp. 541-563. 